\title{
COUPLED DEFECT LEVEL RECOMBINATION IN THE P-N JUNCTION
}

\author{
Juraj RACKO * Miroslav MIKOLÁŠEK * - Peter BENKO * \\ - Ondrej GALLO * Ladislav HARMATHA * \\ — Ralf GRANZNER ${ }^{* *}$ — Frank SCHWIERZ ${ }^{* *}$
}

\begin{abstract}
The well known Shockley-Read-Hall (SRH) model considers emission and capture processes at defects exhibiting a single level or multiple non-coupled levels in the band gap of the semiconductor. The present paper generalizes the model to the case of two mutually coupled defect levels acting as trapping centres. If the intercenter transition is not considered, the model reduces to the case of two non-coupled levels treated by the SRH model.
\end{abstract}

K e y w or d s: tunneling, coupled levels

\section{THEORY}

The paper considers the existence of lattice defects (electrically active traps) having two coupled defect levels (CDL) in the band gap of the semiconductor between which thermal exchange of free charge carriers takes place. The two coupled capture centres are denoted by indices $a$ and $b$ with corresponding energies $E_{t}^{a}$ and $E_{t}^{b}$. We assume that the deep level $E_{t}^{a}$ lies above $E_{t}^{b}$. The well-known SRH model does not consider this case. The commercially available simulator of electron devices and structures DESSIS already contains a CDL recombination model $[1,2]$ which is, however, relatively complicated.

Our CDL model considers ten exchange processes of free charge carriers between the capture centres and the conduction and valence bands. These processes are schematically shown in Fig. 1.

Each of the ten exchange processes (five capture and five emission processes) is characterized by its escape time. If the unknown occupation probability of a trapping centre is divided by the escape time of a capture process, or the probability of non-occupation by the escape time of an emission process, one obtains the frequency of the particular exchange process. The frequencies of exchange processes allow to build two equations with two unknown variables, the occupation probabilities of centres $a$ and $b$. Their solution leads to a quadratic equation yielding finally the occupation probabilities. Then, in terms of the ten escape times and of the evaluated occupation probabilities of centres $a$ and $b$ one can correctly define the $\mathrm{SRH}$ and CDL recombination rates contained in the continuity equations [3]. The quasi-static continuity equations for electrons and holes can be written as

$$
\begin{aligned}
\frac{1}{q} \frac{\mathrm{d} J_{\mathrm{D}}^{e}(x)}{\mathrm{d} x} & =U_{\mathrm{SRH}}(x)+U_{\mathrm{CDL}}^{e}(x), \\
-\frac{1}{q} \frac{\mathrm{d} J_{\mathrm{D}}^{h}(x)}{\mathrm{d} x} & =U_{\mathrm{SRH}}+U_{\mathrm{CDL}}^{h}(x),
\end{aligned}
$$

where $J$ represents the current density, $U$ stands for the generation-recombination rates, $x$ is the coordinate, $q$ is the electron charge and the indices have their obvious meanings.

In our model, the standard SRH electron and hole generation-recombination rates are given as

$U_{\mathrm{SRH}}=\left\{\left(\frac{\tau_{\mathrm{S}}^{a}}{\tau_{\mathrm{Re}}^{a} \tau_{\mathrm{Rh}}^{a}}-\frac{\tau_{\mathrm{S}}^{a}}{\tau_{\mathrm{Ge}}^{a} \tau_{\mathrm{Gh}}^{a}}\right)+\left(\frac{\tau_{\mathrm{S}}^{b}}{\tau_{\mathrm{Re}}^{b} \tau_{\mathrm{Rh}}^{b}}-\frac{\tau_{\mathrm{S}}^{b}}{\tau_{\mathrm{Ge}}^{b} \tau_{\mathrm{Gh}}^{b}}\right) \frac{N_{t}}{2}\right.$,

where $N_{t}$ is the density of traps and the capture and emission escape times are defined as

$$
\begin{aligned}
& \frac{1}{\tau_{\mathrm{Re}}^{a, b}(x)}=v_{\mathrm{th}}^{e} \sigma_{e}^{a . b} n(x), \\
& \frac{1}{\tau_{\mathrm{Rh}}^{a, b}(x)}=v_{\mathrm{th}}^{h} \sigma_{h}^{a . b} p(x), \\
& \frac{1}{\tau_{\mathrm{Ge}}^{a, b}}=v_{\mathrm{th}}^{e} \sigma_{e}^{a, b} N_{C} \exp \left(-\frac{E_{t}^{a, b}}{k T},\right. \\
& \frac{1}{\tau_{\mathrm{Gh}}^{a, b}}=v_{\mathrm{th}}^{h} \sigma_{h}^{a, b} N_{V} \exp \left(-\frac{E_{g}-E_{t}^{a, b}}{k T} .\right.
\end{aligned}
$$

Here, the thermal electron and hole velocities are $v_{\mathrm{th}}^{e, h}=$ $\sqrt{3 k T / m_{e, h}^{*}}, \sigma_{e}^{a, b}$ and $\sigma_{h}^{a, b}$ are electron and hole capture

\footnotetext{
* Slovak University of Technology, Ilkovičova 3, 81219 Bratislava, Slovakia, juraj.racko@stuba.sk; ${ }^{* *}$ Technical University Ilmenau, PF 98684 Ilmenau, Germany
} 


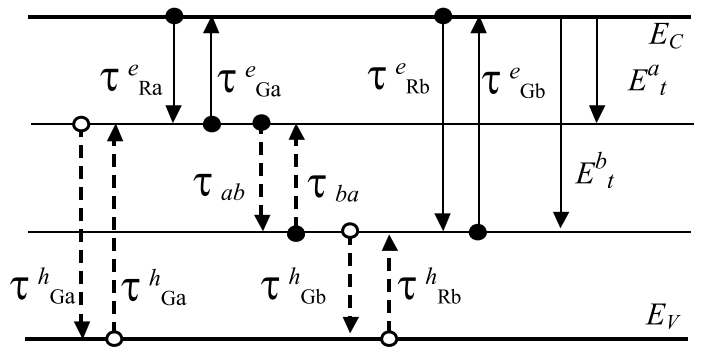

Fig. 1. Ten exchange processes involved in the CDL model. Energies $E_{t}^{a}$ and $E_{t}^{b}$ are taken with respect to the conduction band.

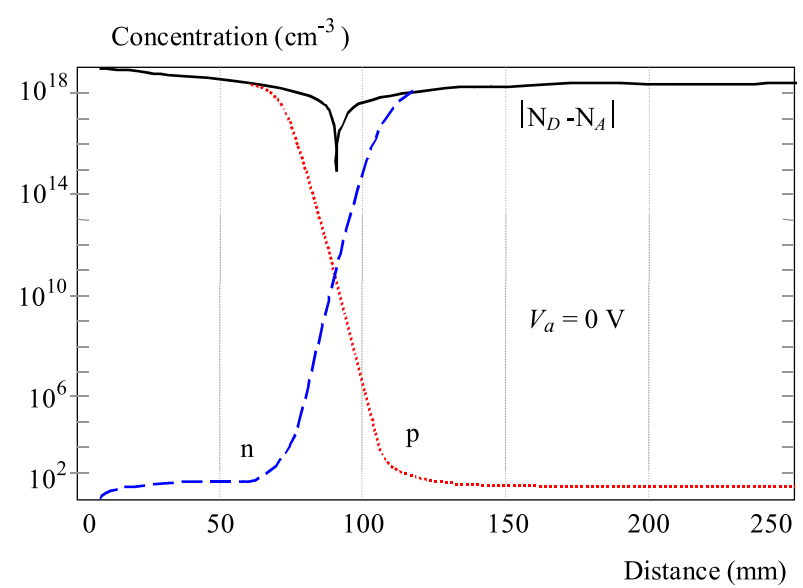

Fig. 2. Concentration profile of simulated PN diode

cross-sections belonging to capture levels $a$ and $b, n(x)$ and $p(x)$ are actual densities of electrons and holes.

The sums of the reciprocal values of the capture and emission escape times are denoted as

$$
\begin{aligned}
& \frac{1}{\tau_{S}^{a, b}}=\frac{1}{\tau_{\mathrm{Re}}^{a, b}}+\frac{1}{\tau_{\mathrm{Ge}}^{a, b}}+\frac{1}{\tau_{\mathrm{Gh}}^{a, b}}+\frac{1}{\tau_{\mathrm{Rh}}^{a, b}}, \\
& \frac{1}{\tau_{\mathrm{Re} G h}^{a, b}}=\frac{1}{\tau_{\mathrm{Re}}^{a, b}}+\frac{1}{\tau_{\mathrm{Gh}}^{a, b}} .
\end{aligned}
$$

On separating the SRH model, the remaining part is the CDL electron and hole recombination model that can be expressed in terms of its CDL generation-recombination rates

$$
\begin{aligned}
U_{\mathrm{CDL}}^{e}(x)= & \left\{\frac{1}{\tau_{\mathrm{Re}}^{a}}+F_{\mathrm{CDL}}^{a}\left(\frac{1}{\tau_{\mathrm{Re}}^{a}}+\frac{1}{\tau_{\mathrm{Ge}}^{a}}\right)\right. \\
& \left.+\frac{1}{\tau_{\mathrm{Re}}^{b}}+F_{\mathrm{CDL}}^{b}\left(\frac{1}{\tau_{\mathrm{Re}}^{b}}+\frac{1}{\tau_{\mathrm{Ge}}^{b}}\right)\right\} \frac{N_{t}}{2}, \\
U_{\mathrm{CDL}}^{h}(x)= & \left\{\frac{1}{\tau_{\mathrm{Gh}}^{a}}+F_{\mathrm{CDL}}^{a}\left(\frac{1}{\tau_{\mathrm{Rh}}^{a}}+\frac{1}{\tau_{\mathrm{Gh}}^{a}}\right)\right. \\
& \left.+\frac{1}{\tau_{\mathrm{Gh}}^{b}}+F_{\mathrm{CDL}}^{b}\left(\frac{1}{\tau_{\mathrm{Rh}}^{b}}+\frac{1}{\tau_{\mathrm{Gh}}^{b}}\right)\right\} \frac{N_{t}}{2},
\end{aligned}
$$

where the coupling functions $F_{\mathrm{CDL}}^{a}$ and $F_{\mathrm{CDL}}^{b}$ are

$$
\begin{aligned}
F_{\mathrm{CDL}}^{a}= & r_{a b}\left(1 \mp D_{a}\right)-\frac{\tau_{S}^{a}}{\tau_{\mathrm{ReGh}}^{b}} \\
& \pm D_{a} \tau_{S}^{a}\left(\frac{1}{\tau_{\mathrm{ReGh}}^{a}}+\frac{1}{\tau_{\mathrm{ReGh}}^{b}}\right), \\
F_{\mathrm{CDL}}^{b}= & -r_{a b}\left(1 \mp D_{b}\right)-\frac{\tau_{S}^{b}}{\tau_{\mathrm{ReGh}}^{a}} \\
& \pm D_{b} \tau_{S}^{b}\left(\frac{1}{\tau_{\mathrm{ReGh}}^{a}}+\frac{1}{\tau_{\mathrm{ReGh}}^{b}}\right) .
\end{aligned}
$$

Functions $r_{a b}$ and $r_{b a}$ are the coupling parameters of the trap levels $E_{t}^{a}$ and $E_{t}^{b}$ expressed as

$$
\begin{aligned}
& r_{a b}=\frac{\frac{\tau_{a b}}{\tau_{S}^{b}}+\frac{\tau_{S}^{a}}{\tau_{S}^{b}}+\exp \left(-\frac{E_{t}^{b}-E_{t}^{a}}{k T / 2}\right)}{1-\exp \left(-\frac{E_{t}^{b}-E_{t}^{a}}{k T / 2}\right)}, \\
& r_{b a}=\frac{\frac{\tau_{a b}}{\tau_{S}^{a}}+1+\frac{\tau_{S}^{b}}{\tau_{S}^{a}} \exp \left(-\frac{E_{t}^{b}-E_{t}^{a}}{k T / 2}\right)}{1-\exp \left(-\frac{E_{t}^{b}-E_{t}^{a}}{k T / 2}\right)},
\end{aligned}
$$

where $\tau_{a b}$ is the coupled escape time defined as

$$
\frac{1}{\tau_{a b}}=\left(v_{\mathrm{th}}^{e} \frac{\sigma_{e}^{a} \sigma_{e}^{b}}{\sigma_{e}^{a}+\sigma_{e}^{b}}+v_{\mathrm{th}}^{h} \frac{\sigma_{h}^{a} \sigma_{h}^{b}}{\sigma_{h}^{a}+\sigma_{h}^{b}}\right) N_{t} .
$$

Coefficients of the quadratic equations $A_{a}, A_{b}, B_{a}, B_{b}$, $C_{a}$ and $C_{b}$ are given by

$$
\begin{aligned}
A_{a}= & \frac{\tau_{S}^{b}}{\tau_{S}^{a}} \frac{1}{\tau_{a b}}\left(1-\exp \frac{E_{t}^{a}-E_{t}^{b}}{k T / 2}\right) \\
A_{b}= & -\frac{\tau_{S}^{a}}{\tau_{S}^{b}} \frac{1}{\tau_{a b}}\left(1-\exp \frac{E_{t}^{a}-E_{t}^{b}}{k T / 2}\right), \\
B_{a}= & \frac{1}{\tau_{S}^{a}}+\frac{1}{\tau_{a b}}+\frac{\tau_{S}^{b}}{\tau_{S}^{a}} \frac{1}{\tau_{a b}} \exp \frac{E_{t}^{a}-E_{t}^{b}}{k T / 2}-\frac{\tau_{S}^{b}}{\tau_{a b}}(1- \\
& \left.\exp \frac{E_{t}^{a}-E_{t}^{b}}{k T / 2}\right)\left(\frac{1}{\tau_{\mathrm{Re}}^{a}}+\frac{1}{\tau_{\mathrm{Gh}}^{a}}+\frac{1}{\tau_{\mathrm{Re}}^{b}}+\frac{1}{\tau_{\mathrm{Gh}}^{b}}\right) \\
B_{b}= & \frac{1}{\tau_{S}^{b}}+\frac{1}{\tau_{a b}} \exp \frac{E_{t}^{a}-E_{t}^{b}}{k T / 2}+\frac{\tau_{S}^{a}}{\tau_{S}^{b}} \frac{1}{\tau_{a b}}+\frac{\tau_{S}^{a}}{\tau_{a b}}(1- \\
C_{a}= & -\left\{\frac{1}{\tau_{\mathrm{Re}}^{a}}+\frac{1}{\tau_{\mathrm{Gh}}^{a}}+\frac{E_{S}^{a}}{\tau_{a b}^{b}}\left(\frac{1}{\tau_{\mathrm{Re}}^{a}}+\frac{1}{\tau_{\mathrm{Gh}}^{a}}+\frac{1}{\tau_{\mathrm{Gh}}^{a}}+\frac{1}{\tau_{\mathrm{Gh}}^{b}}\right)\right. \\
C_{b}= & \left.-\left\{\frac{1}{\tau_{\mathrm{Re}}^{b}}+\frac{1}{\tau_{\mathrm{Re}}^{b}}+\frac{1}{\tau_{\mathrm{Gh}}^{b}}\right) \exp \frac{E_{t}^{a}-E_{t}^{b}}{k T / 2}\right\} \\
& \left.\left.\frac{1}{\tau_{a b}^{b}}+\frac{1}{\tau_{\mathrm{Ge}}^{a}}+\frac{1}{\tau_{\mathrm{Re}}^{b}}+\frac{1}{\tau_{\mathrm{Gh}}^{b}}\right)\right\} \\
& \frac{1}{\tau_{\mathrm{Ge}}}+20
\end{aligned}
$$

and the discriminants of the quadratic equations are

$$
D_{a, b}=\sqrt{1-\frac{4 A_{a, b} C_{a, b}}{B_{a, b}^{2}}} .
$$




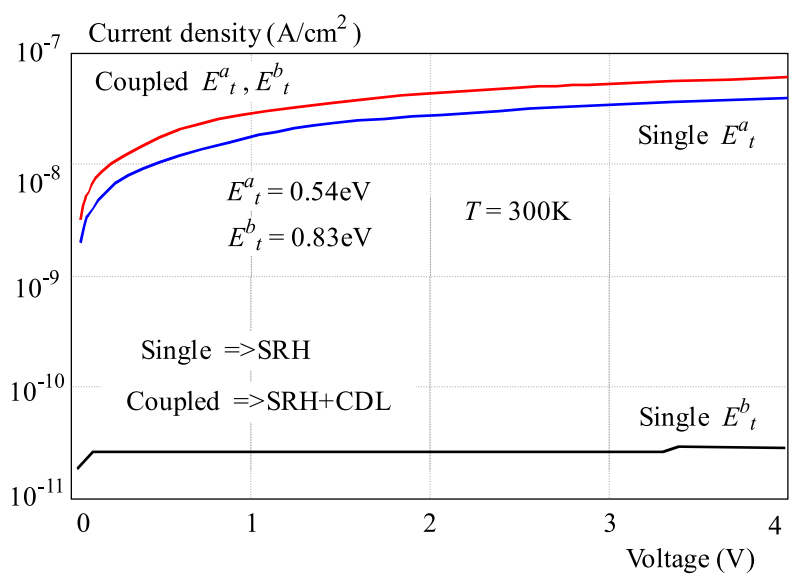

Fig. 3. Comparison of our CDL model with single SRH model of a PN diode contaminated by gold

As long as we do not consider tunnelling of free charge carriers between the trap centres and the conduction or valence band, thus the trap assisted tunnelling, it must hold $U_{\mathrm{CDL}}^{e} \equiv U_{\mathrm{CDL}}^{e}$. This identity expresses the fact that the change in the amount of electrons in the conduction band due to generation and recombination is the same as the change in the amount of holes in the valence band. This is why it is enough to compute one generationrecombination rate only, either $U_{\mathrm{CDL}}^{e}$ or $U_{\mathrm{CDL}}^{h}$, trying to minimize the rounding error. It should be noted that the formulae for $U_{\mathrm{CDL}}^{e}$ and $U_{\mathrm{CDL}}^{h}$ hold only if $E_{t}^{a} \neq E_{t}^{b}$.

\section{SIMULATION RESULTS}

The new CDL recombination model was employed to simulate a PN diode prepared on a (111)-oriented phosphorous-doped silicon substrate $\left(N_{D}=2.5 \times 10^{18} \mathrm{~cm}^{-3}\right)$ by boron diffusion from an infinite source with surface concentration $N_{A}=10^{19} \mathrm{~cm}^{-3}$. The concentration profile $\left|N_{D}-N_{A}\right|$ of the simulated PN diode in thermodynamic equilibrium along with distributions of free electrons and holes are shown in Fig. 2.

Figure 3 displays the simulated reverse $I-V$ curve of the PN diode contaminated by gold which forms two bands of traps, one of acceptor type with a distance of $E_{t}^{a}=0.54 \mathrm{eV}$ from the conduction band edge, and one of donor type with its peak lying at a distance of $E_{t}^{b}=$ $0.83 \mathrm{eV}$ from the conduction band edge, with the same effective cross sections $\sigma_{e, h}^{a, b}=5 \times 10^{-15} \mathrm{~cm}^{2}$. The concentration of gold was assumed to be $N_{t}=5 \times 10^{14} \mathrm{~cm}^{-3}$. Simulations reveal that if the CDL effect is not considered, the donor level $E_{t}^{b}$ has no effect upon the $I-V$ curves of the PN diode. However, if we assume coupling between the deep levels $E_{t}^{a}$ and $E_{t}^{b}$, the impact of the donor level $E_{t}^{b}$ is significant.

Figure 4 shows the reverse $I-V$ characteristics taking into account the CDL effect, with effective cross section $\sigma_{e, h}^{a, b}=5 \times 10^{-15} \mathrm{~cm}^{2}$, constant $E_{t}^{a}=0.55 \mathrm{eV}$ and various $E_{t}^{b}=0.83,0.69,0.54,0.39$ and $0.24 \mathrm{eV}$.

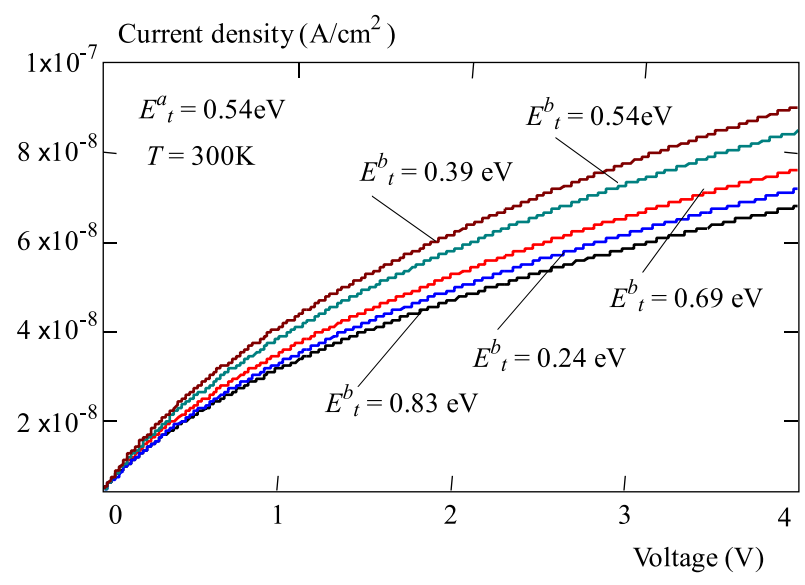

Fig. 4. Reverse I-V characteristics with $\mathrm{CDL}$ effect with $E_{t}^{a}=$ $0.55 \mathrm{eV}$ and various $E_{t}^{b}$

\section{CONCLUSION}

As mentioned previously, the presented model assumes $E_{t}^{a} \neq E_{t}^{b}$. The extreme case, when $E_{t}^{a} \cong E_{t}^{b}$, results in $U_{\mathrm{CDL}}^{e}=U_{\mathrm{CDL}}^{e}=U_{\mathrm{SRH}}$. In other words, the right side of continuity equations (1) and (2) reduces to $U_{\mathrm{SRH}}+U_{\mathrm{CDL}} \cong 2 U_{\mathrm{SRH}}$. In the other extreme case, when $E_{t}^{a}-E_{t}^{b} \cong E_{g}$, thus when the distance between the trap levels $E_{t}^{a}$ and $E_{t}^{b}$ is big and approaches the band gap $E_{g}$, the contributions of both $\mathrm{SRH}$ capture and emission as well as of the CDL effect are negligible, $U_{\mathrm{SRH}}+U_{\mathrm{CDL}} \cong 0$. It is obvious that the CDL effects has a stronger influence in wide gap semiconductors, particularly if dopants or defects exhibit more than two coupled levels in the forbidden band. A more accurate model that is currently under development takes into account that the levels are broadened into band of traps due to multiphonon excitation.

\section{Acknowledgement}

The work has been conducted in the Centre of Excellence CENAMOST (VVCE-0049-07), and supported by the Slovak Research and Development Agency (projects APVV-0133-07) and by the Scientific Grant Agency of the Ministry of Education of the SR (projects VEGA 1/0601/10 and VEGA 1/0507/09).

\section{REFERENCES}

[1] ISE Integrated System Engineering, Release 7.0, Volume 4a (1995), p. 12215.

[2] SCHENK, A.-KRUMBEIN, U.: Coupled Defect Recombination: Theory and Application to Anomalous Diode Characteristics, J. Appl. Phys. 78 (1995), 3185.

[3] RACKO, J.: Unpublished results.

Received 12 February 2011

Juraj RACKO was born in Trenčín in 1953, graduated in experimental physics in 1976 and received his $\mathrm{PhD}$ in electronics and vacuum technology, both from STU, in 1995. At 
present a senior scientist with the Institute of Electronics and Photonics, STU Bratislava, working in modelling and simulation of electron devices and structures.

Miroslav MIKOLÁŠEK was born in Myjava (Slovakia), in 1983. He carried out research in the area of microfluidic circuits at the Johannes Kepler University in Linz, Austria, and based on this work he received his Master degree in electronics from the Slovak University of Technology, Bratislava, in 2007. At present he works at the Institute of Electronics and Photonics, Slovak Technical University in Bratislava. Main interests of his research include simulation and diagnostics in the field of solar cells based on amorphous silicon.

Peter BENKO received his MSc in Electronics from the Slovak University of Technology in Bratislava in 2005 and his $\mathrm{PhD}$ in Electronics from the Slovak University of Technology in 2009. Since 2009 he has worked as a researcher at the Institute of Electronics and Photonics, STU. Main interests of his research include measurement and diagnostics of semiconductor structures and devices.

Ondrej GALLO was born in Rožňava in 1981, Slovakia. He graduated with a Master degree in Radioelectronics from the Slovak University of Technology, Bratislava, in 2007. He works at the Institute of Computer Science and Mathematics of the Slovak University of Technology, in Bratislava. He is interested in the synthesis of asynchronous digital circuit with using Petri nets.
Ladislav HARMATHA was born in Dobšiná, Slovak Republic, in 1948. In 1971 he graduated from the Faculty of Electrical Engineering, STU Bratislava, in 1984 received the $\mathrm{PhD}$ degree and was appointed associate professor in 1996. In 1971 he joined the staff of the Department of Microelectronics, FEI STU in Bratislava, as a research worker. Since 1988 he has worked as a senior scientist in the field of semiconductor defects engineering. His research is focused on defects in semiconductor structures and their characterization by means of electrical methods (capacitance transient spectroscopy). He is the author of about 60 scientific and technical papers and 26 research reports.

Ralf GRANZNER received the Diploma in electrical engineering from Technische Universität (TU) Ilmenau, Germany, in 2000. Since then he has been with the RF and Nano Device Research Group, Fachgebiet Festkrperelektronik, TU Ilmenau. His current research work focuses on modelling and simulation of extremely scaled MOSFETs and ultrafast SiGe HBTs.

Frank SCHWIERZ received the Diploma, Dr-Ing and Dr habil degrees from Technische Universität (TU) Ilmenau, Germany, in 1982, 1986, and 2003, respectively. He is currently Privatdozent and Head of the RF and Nano Device Research Group, Fachgebiet Festkörperelektronik, TU Ilmenau. His current research interests include advanced semiconductor device simulation, very high-speed transistors and novel device and material concepts for future ULSI and RF transistor generations.

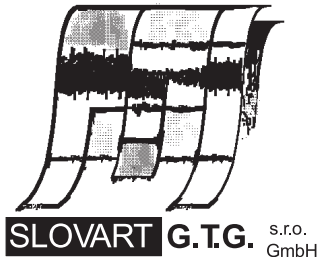

EXPORT - IMPORT
EXPORT - I M P OR T

of periodicals and of non-periodically printed matters, books and CD-ROMs

Krupinská 4 PO BOX 152, 85299 Bratislava 5, Slovakia tel: ++421 263839 472-3, fax: ++421263839485 info@slovart-gtg.sk; http://www.slovart-gtg.sk

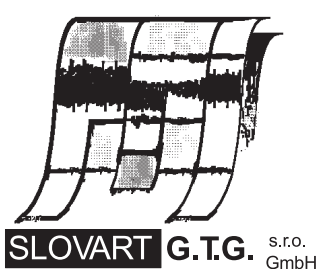

EXPORT - IMPORT 